\title{
PV diagrams for the maser emission from a Keplerian ring
}

\author{
L. Uscanga ${ }^{1}$, J. Cantó ${ }^{2}$ and A. C. Raga ${ }^{3}$ \\ ${ }^{1}$ Centro de Radioastronomía y Astrofísica, UNAM, Ap. 3-72, 58089 Morelia, Mich., Mexico \\ email: l.uscanga@astrosmo.unam.mx \\ ${ }^{2}$ Instituto de Astronomía, UNAM, Ap. 70-264, 04510 México, D.F., Mexico \\ ${ }^{3}$ Instituto de Ciencias Nucleares, UNAM, Ap. 70-543, 04510 México, D.F., Mexico
}

\begin{abstract}
We study the maser emission arising from a thin, planar, gaseous ring in Keplerian rotation around a central mass when it is observed edge-on. Assuming that the absorption coefficient within the amplifying ring is a decreasing function of distance from the central mass (i.e., $\kappa=\kappa_{0} r^{-q}$ ), we calculated position-velocity (PV) diagrams for the most intense maser features using different values of the exponent $q$. We found that, depending on the value of $q$, these diagrams can be qualitatively different.
\end{abstract}

Keywords. galaxies: individual (NGC 4258) — galaxies: nuclei — masers

\section{Introduction}

The spatial distribution and line-of-sight velocities of the systemic and high-velocity water masers in NGC 4258 trace a thin molecular ring in Keplerian rotation around a massive black hole of $3.6 \times 10^{7} \mathrm{M}_{\odot}$ seen nearly edge-on (Miyoshi et al. 1995). The PV diagram for the maser emission shows distinct Keplerian orbits defined by the highvelocity maser emission, as well as a line traced by the systemic maser emission. The systemic masers lie within a narrow range of radii, on the near side inner edge of the ring, while the high-velocity masers are located near the ring diameter. Previously, Watson \& Wallin (1994) showed that the maser emission from a rapidly rotating, thin Keplerian ring viewed edge-on can reproduce the general features of the observed $22 \mathrm{GHz}$ radiation of this galaxy. However, their assumption of a uniform absorption coefficient within the amplifying ring results in a PV diagram for the most intense masers that is qualitatively different from the observed one. Here we show that this discrepancy can be resolved if the absorption coefficient decreases with distance from the central mass.

\section{Model and results}

We consider a masering Keplerian ring seen edge-on. The masering gas is located between $R_{0}$ and $R$, the inner and outer radii of the ring, respectively. We assume that the absorption coefficient is $\kappa=\kappa_{0} r^{-q}$. Distances are measured in units of $R$, and velocities are measured in units of $v_{\text {out }}$, the rotation velocity at the outer edge of the ring. For the case of an unsaturated maser and neglecting the spontaneous emission, the intensity of the maser radiation from a line of sight with impact parameter $y$ at a velocity $v_{r}$ is

$$
I\left(v_{r}, y\right)=I_{0} e^{\tau\left(v_{r}, y\right)},
$$



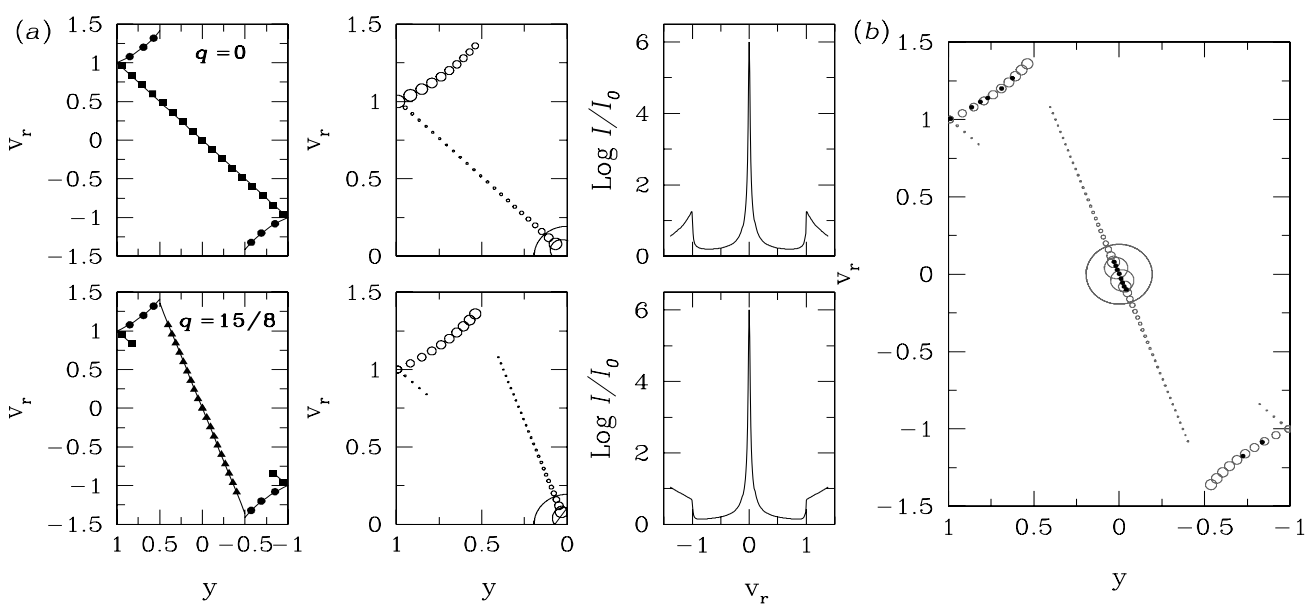

Figure 1. (a) Results of model with $q=0,15 / 8$. Left panels: PV diagrams for the maser emission peak. The filled squares, circles, and triangles represent the strongest maser emission which is coming from the outer edge, mid-line, and inner edge of the ring, respectively. The straight lines or curves represent the velocity dependencies of the regions where this emission arises. Central panels: PV diagrams for the maser emission peak, only positive velocities are shown. The radii of the open circles are proportional to the maximum maser intensity at each position and velocity. Right panels: the logarithm of the ratio between the maximum intensity and the background intensity as a function of the velocity. (b) Comparison between the calculated PV diagram for the maser emission peak using $q=15 / 8$ and the PV diagram delineated by the water masers observed in NGC 4258. The dots represent the observed maser spots (Miyoshi et al. 1995). We subtracted the ring systemic velocity of $476 \mathrm{~km} \mathrm{~s}^{-1}$ from the observed LSR velocity of the maser spots in order to compare the observed PV diagram with the modelled one. The positions and velocities are in units of the outer radius of the ring $(8 \mathrm{mas})$ and the rotation velocity at the outer edge of the ring $\left(770 \mathrm{~km} \mathrm{~s}^{-1}\right)$, respectively (Uscanga et al. 2007).

where the optical depth or gain along the line of sight is given by

$$
\tau\left(v_{r}, y\right)=\kappa_{0} \int\left(x^{2}+y^{2}\right)^{-q / 2} \exp \left[\frac{-\left(v-v_{r}\right)^{2}}{\Delta v_{D}^{2}}\right] d x .
$$

We numerically solved equations (2.1) and (2.2) and calculated the $y$-positions (impact parameters) of maximum maser intensity for each specific value of $v_{r}$. When we found two local maxima, we kept both. Then we constructed the PV diagrams using the positions of the observer's line of sight with maximum emission at each velocity (Figure 1).

\section{Conclusions}

A comparison with the PV diagram observed for the water maser emission in the nucleus of the galaxy NGC 4258 indicates that the exponent $q$ must be higher than 1 to have significant emission coming from the inner edge of the amplifying ring and hence explain the form of the PV diagram. According to our simple model, this implies that the absorption coefficient must depend on the radius of the ring as a decreasing function.

\section{References}

Miyoshi, M., Moran. J., Herrnstein, J., et al. 1995, Nature, 373, 127

Uscanga, L., Cantó, J., \& Raga, A. C. 2007, ApJ, in press

Watson, W. D., \& Wallin, B. K. 1994, ApJ (Letters), 432, L35 